\title{
PRÄVENTION
}

\section{Gesunder Hausarzt, gesunde Patienten}

Wie gut ein Arzt für die eigene Gesundheit sorgt, zeigt sich auch an seinen Patienten. Bei der Inanspruchnahme von Präventionsmaßnahmen besteht eine positive Korrelation zwischen Arzt- und Patientenverhalten. Diesen Zusammenhang konnten Ärzte aus Vancouver und Tel Aviv jetzt anhand von Daten der größten Krankenversicherung Israels objektivieren. Als Maß für das Gesundheitsverhalten diente die dokumentierte Teilnahme an empfohlenen Screening-Untersuchungen (Mammografie, Hämoccult oder Koloskopie, LDL-Messung, Blutdruckmessung) und Impfungen (gegen Influenza und Pneumokokken). Der
Vergleich ergab, dass Patienten die empfohlenen Screening-Untersuchungen und Impfungen signifikant häufiger absolvierten, wenn ihr Arzt die jeweilige Präventionsmaßnahme selbst auch befolgte. Der Unterschied zwischen Patienten von adhärenten und nicht adhärenten Ärzte war für jede der erfassten Vorsorgemaßnahmen signifikant. Besonders deutlich zeigte sich die Differenz bei der Influenza-Impfung: 49,1\% der Patienten von geimpften Ärzten, aber nur 43,2\% von nicht geimpften Ärzten hatten sie erhalten.

CMAJ 2013; online 8. April; doi:10.1503/cmaj.121028

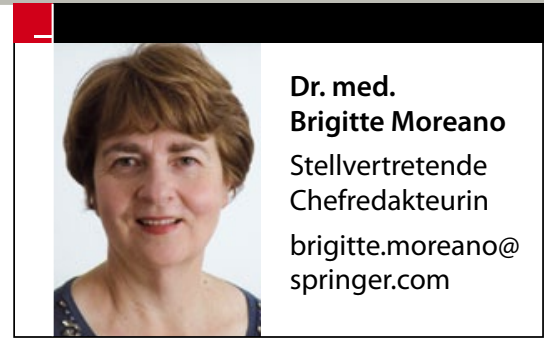

\section{DEMENZRISIKO}

\section{Diabetiker erwischt es zwei Jahre früher}

Diabetiker erkranken gut zwei Jahre früher an einer Demenz als Nicht-Diabetiker. Ihre Überlebenszeit nach der Demenzdiagnose ist ebenfalls verkürzt. Eine bevölkerungsbasierte Studie aus Australien zeigt, dass sich eine vaskuläre Demenz im Schnitt bei Diabetikern fast drei Jahre und eine AlzheimerDemenz gut ein Jahr früher als bei Stoffwechselgesunden manifestiert.

Ausgewertet wurden die Daten von 25000 Bewohnern Westaustraliens, die zwischen 1990 und 2005 eine Demenzdiagnose erhalten hatten. Knapp drei Viertel der demenzkranken Patienten starben im Beobachtungszeitraum. Bei Diabetikern trat der Tod durchschnittlich 2,6 Jahre früher ein als bei Nicht-Diabetikern.

American Journal of Epidemiology Advance Access published March 28, 2013; DOI: 10.1093/aje/kws387

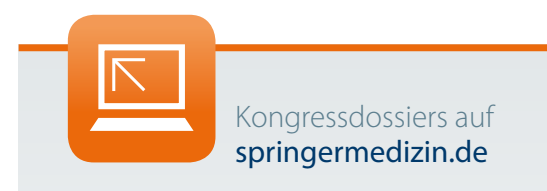
den mit intensivierter Insulintherapie und 17\% der Diabetiker mit konventioneller Insulintherapie ihre Fahrerlaubnis innerhalb des Studienzeitraums verloren.

Diabetic Medicine 2013; online 28. Februar; doi: 10.1111/dme.12075

die Zahl der Hypoglykämieepisonden bei 1441 Patienten untersucht. Knapp ein Drittel der Diabetiker erlebte innerhalb von zwölf Monaten mehr als eine schwere Hypoglykämie. Nach den neuen Richtlinien erlaubnis nicht zu erteilen bzw. zu entziehen. In einer britischen Studie wurde nun untersucht, wie viele Typ-1-Diabetiker nach der neuen Regelung mit einem FührerZeitraum von sechseinhalb Jahren wurde springermedizin.de

Zwei umfangreiche Dossiers von den großen Frühjahrskongressen stehen online für Sie bereit. Zum Wiesbadener Internistenkongress geht es unter: >www.springermedizin.de/ dgim-2013, zur Jahrestagung der Deutschen Gesellschaft für Kardiologie gelangen Sie auf: >www.springermedizin.de/ dgk-2013 